Medicine Updates

Faculty of medicine

October 2020,volume 3, https://muj.journals.ekb.eg

dean@med.psu.edu.eg

vice_dean_postgraduate@med.psu.edu.eg

DOI $\quad 10.21608 /$ muj.2020.41090.1023

\title{
Effect of N-Acetylcysteine on Total Oxidant Status in Children With $\beta$-Thalassemia Major
}

Amany A. Ahmed Bany Sweif Specialized Hospital.

Mohamed H. Meabed Pediatrics Department, Faculty of Medicine, Beni-Suef University, Egypt

Yasmeen A. Mohamed Pediatrics Department, Faculty of Medicine, Beni-Suef University, Egypt

Rehab M. Abd-Elkareem Pediatrics Department, Faculty of Medicine, Beni-Suef University, Egypt

\begin{abstract}
$\underline{\text { Abstract }}$
ABSTRACT: $\beta$-Thalassemias are due to $\beta$-globin gene mutation leading to the absence or reduction of $\beta$-globin chains formation. Numerous studies are showing the rising of oxidative stress in $\beta$-thalassemia major patients. The study aims to evaluate the effects of $\mathrm{N}$-acetylcysteine (NAC) on total oxidant status and $\mathrm{Hb}$ levels in children with $\beta$-thalassemia major. This study is a randomized clinical trial in the department of pediatrics, Beni-Suef University during the period between May 2019 and October 2019. The subjects of the study were beta-thalassemia major, the study was conducted on 44 patients that were divided into two groups, 22 patients took NAC at a dose of $10 \mathrm{mg} / \mathrm{kg}$ (solvymist syrup or, NAC sachet) orally, for 3 months, and the second group as a non-treatment group. Article results show a significant increase in HB for
\end{abstract}


the treatment group between form the first measurement until the $3^{\text {rd }}$ month. There was a significant decrease between Total Oxidant Status before and after three months in the treatment group. Therefore, NAC may be effective in reducing serum Total oxidant status and increase pre-transfusion $\mathrm{Hb}$ levels in children with $\beta$-thalassemia major.

Keywords: TOS, Acetylcysteine, B-Thalassemia, Oxidant, Children, Randomized

\section{Abbreviations:}

$\mathrm{Hb}$ : hemoglobin

TOS: Total oxidant status

NAC: $\mathrm{N}$-acetylcysteine

SD: Standard deviation s

\section{INTRODUCTION}

$\beta$-thalassemias are characterized by the presence of mutations on the $\beta$-globin gene, resulting in the absence or reduced synthesis of $\beta$-globin chains of the hemoglobin (Hb) tetramer (Cao \& Galanello, 2010). Thalassemias are a group of inherited hematological disorders caused by defects in the synthesis of one or more of the hemoglobin chains (Muncie Jr \& Campbell, 2009). Imbalances of globin chains cause hemolysis and impair erythropoiesis. Beta thalassemia major causes hemolytic anemia, poor growth, and skeletal abnormalities during infancy. Affected children will require regular lifelong blood transfusions. (Origa, 2017).Transfusion-dependent patients will develop iron overload and require chelation therapy to remove excess iron. Persons with thalassemia should be referred for preconception genetic counseling. Persons with beta-thalassemia major often die from cardiac complications of iron overload by 30 years of age (Muncie Jr \& Campbell, 2009). Early and regular blood transfusion therapy decreases the complications of severe anemia and prolongs survival. In the long term, however, the beneficial effects of transfusions are limited by the organ damage resulting from cumulative iron burden (Prati, 2000) When body iron is excessive, increased non-transferrin-bound iron induces free radicals (Hershko, 2010). Reactive oxygen species cause damage to macromolecules, oxidation of amino acid side, and polypeptide chains with the result of DNA damage (Zastawny et al., 1995). Therefore, we need iron chelation in the management of this fatal disease. The choice of effective chelation therapy protocols that decrease the iron load of patients to near ordinary physiological ranges is needed (Kolnagou, Economides, Eracleous, \& Kontoghiorghes, 2008). Antioxidants help to eradicate toxic oxygen radicals (Kolnagou et al., 2008). NAC entered the cells and converted to 1-cysteine, which gives the aminothiol Glutathione (GSH). GSH is a major 
intracellular antioxidant (Faintuch, Aguilar, \& Nadalin, 1999). It is said that treatment by combinations of antioxidants such as NAC plus iron chelators could mollify the damaging effects of Reactive Oxygen Species (ROS) (Rachmilewitz, WEIZER-STERN et al. 2005). In order to fulfill the objectives of this study.

\section{AIM OF THE STUDY}

The present study aims to evaluate the effects of N-acetylcysteine (NAC) on total oxidant status and Hemoglobin $(\mathrm{Hb})$ levels in children with a $\beta$-thalassemia major within 3 months. The Study type is a randomized clinical trial and setting all patients of the study were subjected to the following after approval of the internal ethical committee:

(1) Careful histories are Age and sex of the patient, Age of patient at $1^{\text {st }}$ diagnosis of the disease, Age of first blood transfusion, Frequency of blood transfusion, history of operations, and Family history.

(2) Complete clinical examination every month

(3) Laboratory investigations are Hemoglobin level, Serum ferritin level measurement before giving NAC and after treatment for 3 months, Liver functions (Alanine transaminase, Aspartate transaminase) before giving NAC and after treatment for 3 months, and Serum level of total oxidant status before giving NAC and after treatment for 3 months.

\section{Subjects and Methods:}

Subjects: This study was performed in an outpatient hematology pediatric clinic in Beni-Suef university hospital. Subjects of the study were beta-thalassemia major patients with age ranged from 2 to 13 years on iron chelators, transfusion-dependent, and not suffering from any complication (respiratory, cardiac, or endocrine). The criterion for sample size is a sample of $\mathbf{4 4}$ subjects. The subject of the study was divided into two groups: The first group Included 22 patients with beta-thalassemia major received NAC at a dose of $10 \mathrm{mg} / \mathrm{kg} / \mathrm{day}$ (solvymist syrup or, NAC sachet) orally, for 3 months and the second group included 22 patients with beta-thalassemia major did not receive NAC

The inclusion criteria are patients receiving iron chelators and transfusiondependent thalassemia patients. The exclusion criteria are the patients are not receiving an iron chelator and on regular transfusion. Patient with any complication (respiratory, cardiac or endocrine) Operational design: The study had been explained to all participants with the informed written consent by the parents of the patients after approval of the local ethical committee. 
Protocol Approval by Ethical Committee Before the beginning of the study and in accordance with the local regulation followed the protocol and all corresponding documents were declared for Ethical and Research approval by Beni-Suef university hospital and Institutional Review Broad (IRB).

\section{METHODS:}

All patients of the study were subjected to the following after approval of internal ethical committees: (1) History taking stressing upon the Age and sex of the patient, Age of patient at $1^{\text {st }}$ diagnosis of the disease, Age of first blood transfusion, Frequency of blood transfusion and history of operations and Family history. (2) Complete clinical examination. (3). The general examination includes the Clinical picture of thalassemia, vital signs, weight in $(\mathrm{kg})$, and any complications (chest, cardiac or hepatic) and any organometallic. (4) Laboratory investigations including Hemoglobin level, serum ferritin level, liver functions, and Serum level of total oxidant status.

\section{Statistics Methods}

All data are analyzed, represented, and summarized by using appropriate statistical tests using statistical package for the Social Sciences(SPSS) version 25 (IBM Corp., Armonk, NY, USA). Tests of significance (Mann-Whitney test Friedman test, Wilcoxon signed-rank test, Chi-Square $(\chi 2)$ test, and spearman correlation coefficient) were used (Chan, 2003a, 2003b)

\section{RESULTS}

The studying of demographic data of patients in our study shows that the percentage of male patients was $52.3 \%$ and the percentage of female patients was $47.7 \%$. With a mean age of 8.05 and standard deviation $(\mathrm{SD}) \pm 3.35$ years and the percentage of patients with positive family history was $50 \%$ for total patients of 44 . The percentage of positive consanguinity of patients was 50\%. The percentage of patients with hepatomegaly was $84.1 \%$ of the 44 patients. The percentage of patients with splenomegaly was $61.4 \%$, the percentage of patients without splenomegaly was $18.20 \%$ and the percentage of patients with splenectomy was $20.5 \%$ given by table (1). 
Table (1): Number and percentage of splenomegaly or Splenectomy in studied patients

\begin{tabular}{|l|l|l|l|}
\hline Splenomegaly & Yes & No & Splenectomy \\
\hline Number & 27 & 8 & 9 \\
\hline Percentage & $61.4 \%$ & $18.20 \%$ & $20.5 \%$ \\
\hline
\end{tabular}

Table (2): Comparison between two groups concerning laboratory data:

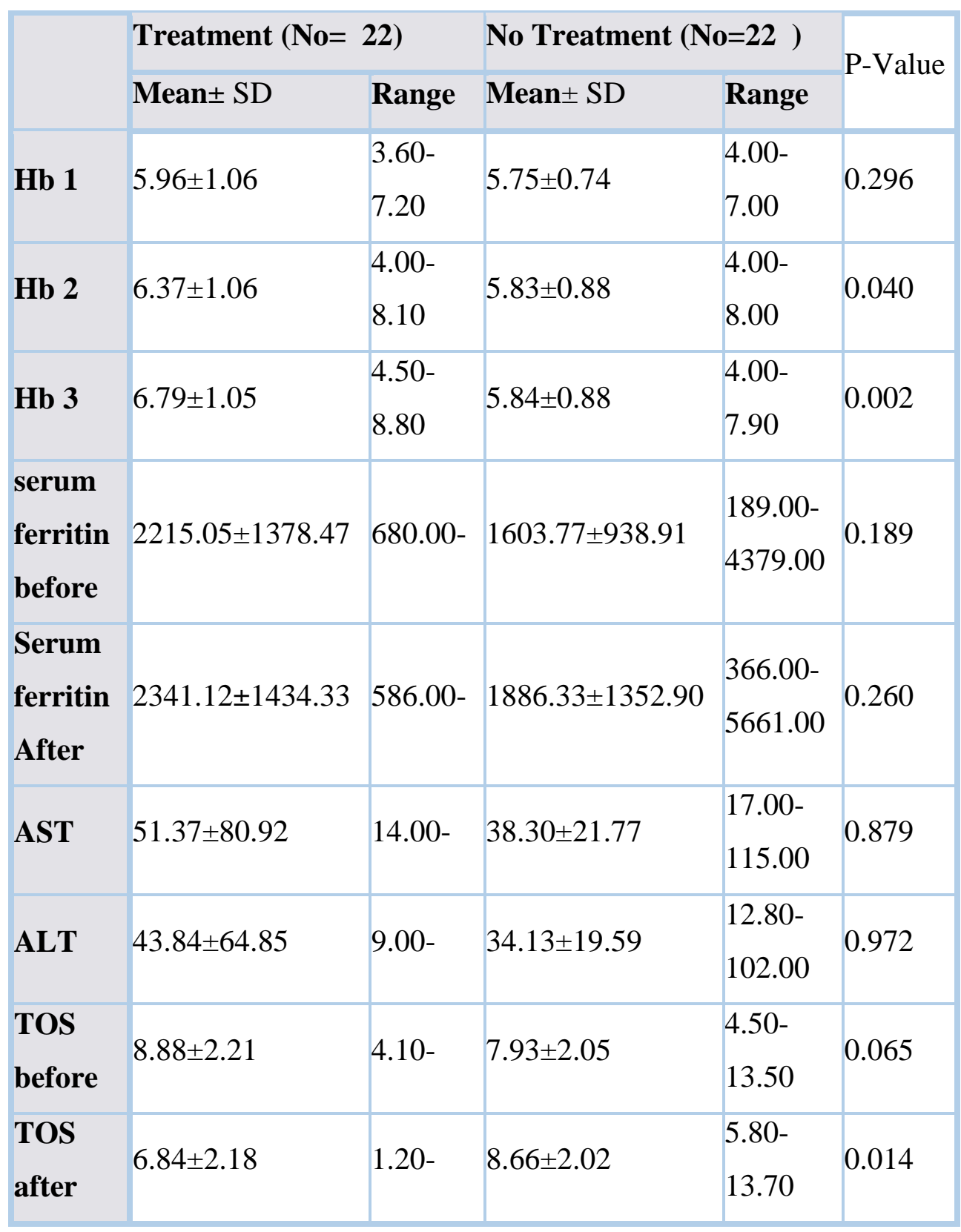

The $\mathrm{Hb} 1$ represents the level of hemoglobin measured at the end of the first month after starting the clinical trial , $\mathrm{Hb} 2$ represents level of hemoglobin at the end of the second month, $\mathrm{Hb} 3$ represents level of hemoglobin at the end of the third month, serum ferritin before represent level of ferritin at the beginning of the clinical trial for 
both groups, Serum ferritin after represents level of ferritin after 3 months, Total Oxidant Status (TOS) before represents the level of TOS at the beginning of the clinical trial for both group and TOS after represents the level of TOS after 3 months of the clinical trial. $\mathrm{Hb} 1$ in the treatment group ranged from 3.60 to $7.20(\mathrm{~g} / \mathrm{dl})$ with a mean of $5.96 \pm 1.06(\mathrm{SD})$ and $\mathrm{Hb} 1$ in the non-treatment group ranged from 4.00 to $7.00(\mathrm{~g} / \mathrm{dl})$ with a mean of $5.75 \pm 0.88(\mathrm{SD})$. The difference was not statistically significant, the p-value was 0.296 .

As shown in the table(2): $\mathrm{Hb} 2$ in the treatment group ranged from 4.00 to $8.10(\mathrm{~g} / \mathrm{dl})$ with a mean of $6.37 \pm 1.06(\mathrm{SD})$ and $\mathrm{Hb} 2$ in the non-treatment group ranged from 4.00 to $8.00(\mathrm{~g} / \mathrm{dl})$ with a mean of $5.83 \pm 0.88(\mathrm{SD})$. It shows good significant p-value (0.04) which indicting an increase of $\mathrm{Hb}$ in case of treatment with NAC and $\mathrm{Hb} 3$ in the treatment group ranged from 4.50 to $8.80(\mathrm{~g} / \mathrm{dl})$ with a mean of $6.79 \pm 1.05(\mathrm{SD})$ and $\mathrm{Hb} 3$ in the non-treatment group ranged from 4.00 to 7.90 with a means of $5.84 \pm$ 0.88 (SD). It shows a significant increase as the p-value (0.002) indicting 9increases of $\mathrm{Hb}$ in case of treatment with NAC.

TOS before in the treatment group ranged from 4.10 to 13.00 with a mean of $8.88 \pm$ $2.21(\mathrm{SD})$ and TOS before in the non-treatment group ranged from 4.50 to 13.50 with a mean of $7.93 \pm 2.05$ (SD). TOS before in the 2 group shows difference was not statistically significant, the p-value was $(0.065)$. TOS after in the treatment group ranged from 1.20 to 9.90 with a mean of $6.84 \pm 2.18$ and TOS after in the nontreatment group ranged from 5.80 to 13.70 with a mean of $6.84 \pm 2.18$. TOS after in 2 groups shows difference was not statistically significant, the p-value was (0.014). By comparing between the 2 groups in the $\mathrm{Hb} 2$ level shows improvement in the case of the treatment group.
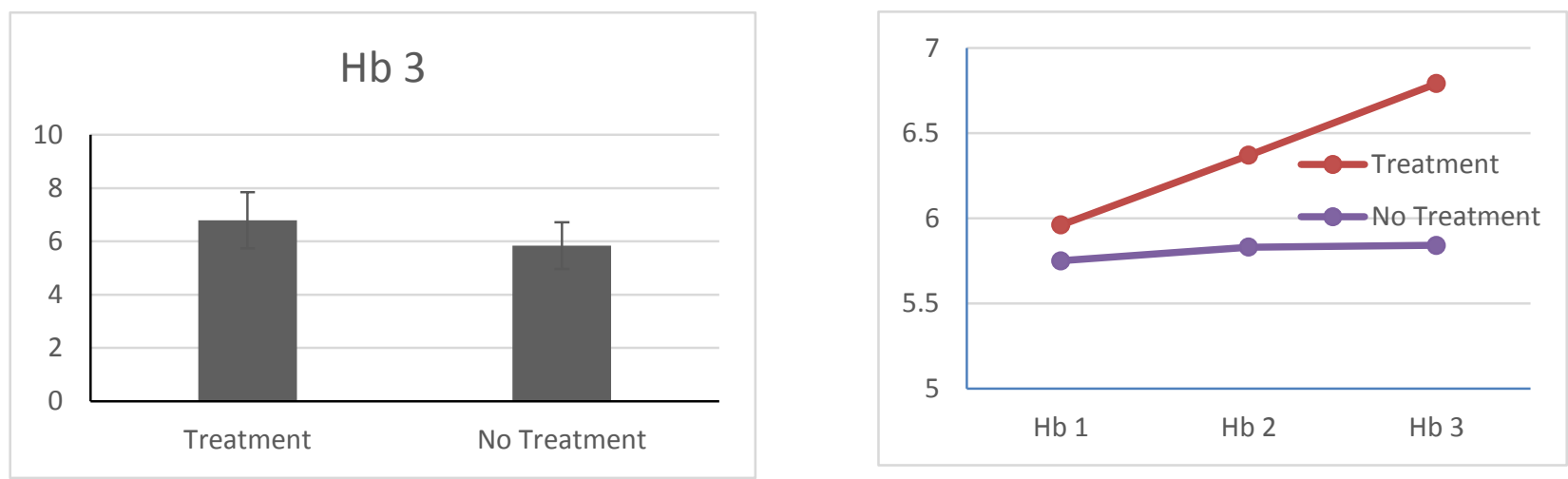
Figure 1: Discrepancy between $\mathrm{Hb}$ level in treatment and non-treatment groups
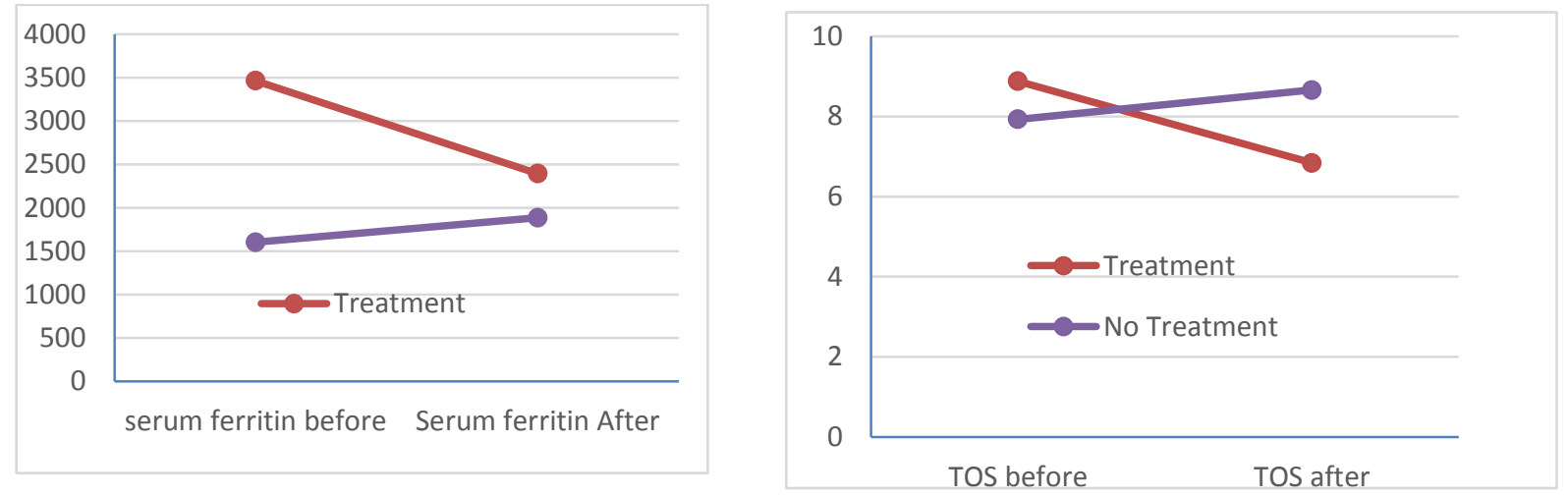

Figure 2: (a) Discrepancy between serum ferritin before and serum ferritin after 3 months in treatment and non-treatment group (b) Discrepancy between TOS

\section{before and after 3 months in treatment and non-treatment group}

Figure 1 shows that $\mathrm{Hb}$ level increased with treatment while its values remained almost constant in the non-treatment group, figure 2 shows a decrease of serum ferritin with treatment after three months and increases in the non-treatment group, figure 3.shows TOS decreased in the treatment group and increased in the nontreatment group. Table (3) shows that there was a significant increase as the p-value < 0.001 in the treatment group between the Hb1, 2, 3 levels. Although a decrease in serum ferritin levels before and after 3 months of treatment there was no significant difference between them. There was a significant decrease between TOS before and TOS after three months of treatment with a p-value (0.001).

In Table (4) shows that in non- treatment group, no significant difference in serum ferritin before, and 3 months after treatment. TOS increased significantly from $7.93 \pm 2.05$ before treatment to $8.66 \pm 2.02$ after 3 months of treatment (p: 0.002 ) 
Table (3): Comparison of laboratory data in the treatment group:

\begin{tabular}{|l|l|l|l|}
\hline \multirow{2}{*}{} & \multicolumn{2}{|l|}{ Treatment(No=22) } & \multirow{2}{*}{ P-value } \\
\cline { 2 - 3 } & Mean \pm SD & Range & \\
\hline Hb 1 & $5.96 \pm 1.06$ & $7.20-3.60$ & \multirow{2}{*}{$<0.001$} \\
\hline Hb 2 & $6.37 \pm 1.06$ & $8.10-4.00$ & \\
\hline Hb 3 & $6.79 \pm 1.05$ & $8.80-4.50$ & \multirow{2}{*}{0.889} \\
\cline { 1 - 3 } serum ferritin before & $3464.5 \pm 1378.47$ & $6249.00-680.00$ & \multirow{2}{*}{0.001} \\
\hline Serum ferritin After & $3293 \pm 1434.33$ & $6000.00-586.00$ & \\
\hline TOS before & $8.88 \pm 2.21$ & $13.00-4.10$ & \\
\hline TOS after & $6.84 \pm 2.18$ & $9.90-1.20$ & \\
\hline
\end{tabular}

Table (4): Comparison between laboratory data in the non-treatment group

\begin{tabular}{|l|l|l|l|}
\hline \multirow{2}{*}{ Hb 1 } & \multicolumn{2}{|c|}{ No Treatment(No=22) } & \multirow{2}{*}{ P-value } \\
\cline { 2 - 3 } & Mean \pm SD & Range & \\
\hline Hb 2 & $5.75 \pm 0.74$ & $7.00-4.00$ & \multirow{2}{*}{0.306} \\
\hline Hb 3 & $5.83 \pm 0.88$ & $8.00-4.00$ & \\
\hline serum ferritin before & $1603.77 \pm 938.91$ & $4379.00-189.00$ & \multirow{2}{*}{0.465} \\
\hline Serum ferritin After & $1886.33 \pm 1352.90$ & $5661.00-366.00$ & \\
\hline TOS before & $7.93 \pm 2.05$ & $13.50-4.50$ & \multirow{2}{*}{0.002} \\
\hline TOS after & $8.66 \pm 2.02$ & $13.70-5.80$ & \\
\hline
\end{tabular}

\section{DISCUSSION}

Thalassemias are due to the reduction or absence of alpha- or beta chains in the hemoglobin (Patpan et al., 2019). The treatment of $\beta$-thalassemia major is blood transfusion to apply normal growth and suppresses ineffective erythropoiesis. Though, regular blood transfusion cause future problems as excess iron. The excess iron is awfully toxic and leads to irreversible organ damage(Mishra \& Tiwari, 2013). Therefore, thalassemia patients have increased oxygen-free radicals. Several studies prove that toxic forms, as non-transferrin-bound iron (NTBI) causes over-reactive oxygen species. Iron is a catalyst in the formation of the hydroxyl radical $(\mathrm{OH})$ that acting as a Fenton reagent (Patpan et al., 2019) . Fenton has other reagents of home chromes that belong to denature ferric proteins, which cause vital organ damage besides the hematopoietic system as well. Cellular damage is linked to iron overload, 
which causes production excess of ROS above the cellular defense abilities. Reactive oxygen species lead to macromolecules damage, lipid peroxidation, oxidation of amino acid and polypeptide chains causing cell death(Cappellini, Cohen, Porter, Taher, \& Viprakasit, 2014). The main objective of this study was to evaluate the effects of NAC on total oxidant status and on $\mathrm{Hb}$ levels in children with $\beta$-thalassemia major. A randomized clinical trial was adopted including $44 \beta$-thalassemia major patients from the regular attendants of the pediatric Hematology Clinic, Beni Suef University Hospital as 22 patients received NAC for 3 months and 22 did not receive NAC. The duration of the study ranged from 3 to 6 months.

Our study showed that the patient's age was $8.05 \pm 3.35$. But in (Ozdemir, Koc, Aycicek, \& Kocyigit, 2014), the mean age of their participants was $9.6 \pm 4.15$. The tested samples in our studies were $47.7 \%$ females and $52.3 \%$ males. They were divided $50 \%$ with family history and the other half without a family history. Positive consanguinity was found in $50 \%$, which indicates the role of genetic counseling in the premarital stage.

Our study shows that $84.1 \%$ of our patients had hepatomegaly, $61.4 \%$ of them had splenomegaly, and $20.5 \%$ of them had a splenectomy. Patpan et al., 2019 reported that most of the patients had beta-thalassemia/hemoglobin E (41 patients, 59.42\%) and most were splenectomies (49 patients, 71.01\%) (Patpan et al., 2019). But Ozdemir et al., 2014 reported that the percentage of splenectomy in the NAC group was $40 \%$ for 10 patients(Ozdemir et al., 2014) .In the study in our hands, the samples are divided for treated and non-treated. Our results are supported by the study of Hamdy et al., 2015 as they reported that $56.7 \%$ of their patients were males, $43.3 \%$ of them were females. $36.6 \%$ of them had a family history and $66.7 \%$ of them had positive consanguinity. In our study female-to-male ratio 21:23 but in Yanpanitch et al., 2015, the female-to-male ratio was 14: 11 in their patients(Hamdy, Mosallam, Jamal, \& Rabie, 2015). In our study, the patient's weight in $\mathrm{kg}$ was about $22.16 \pm$ 6.81 but in Ozdemir et al., 2014, the mean of their weight was $28.5 \pm 11.7 \mathrm{Kg}$ (Ozdemir et al., 2014). In our study, there was no significant difference for treated and non-treated samples regarding age, age of first blood transfusion, frequency of transfusion, and their weight. Our results are supported by the study of Patpan et al., 2019 as they reported that there were no significant effects among studied groups as regards: age and age of first transfusion (Patpan et al., 2019). Our study involved a follow-up of $\mathrm{Hb}$ level every month and in our search no study follows up $\mathrm{Hb}$ level after giving NAC to children with beta-thalassemia major in Egypt. As regard 
comparison between treatment and the non-treatment group, there was a significant difference between them in $\mathrm{Hb} 2$ as p-value 0.040 and also there was a significant difference between them in $\mathrm{Hb} 3$ as a p-value of 0.002 which indicate improvement in the treatment group with NAC. There was a significant increase in treatment group $\mathrm{Hb} 1, \mathrm{Hb} 2$, and $\mathrm{Hb} 3$ with a p-value less than 0.001 . Also in $\mathrm{Hb} 1$ and $\mathrm{Hb} 2$, there was a significant increase with $\mathrm{P}$-value 0.025 . Also in $\mathrm{Hb} 1$ and $\mathrm{Hb} 3$, there was a significant increase as $\mathrm{P}$ value $<0.001$ and a significant increase between $\mathrm{Hb} 2$ and $\mathrm{Hb} 3$ as $\mathrm{P}$ value 0.013 . But in the non-treatment group no significant difference between $\mathrm{Hb} 1$, $\mathrm{Hb} 2$, and $\mathrm{Hb} 3$ as P-value 0.306. This indicates that, hemoglobin improved with NAC for the treatment group and it increased with the follow-up. Our results are in agreement with the study of Ozdemir et al., 2014. As they reported that pretransfusion hemoglobin $(\mathrm{Hb})$ levels were significantly increased after 3 months $(\mathrm{p} \leq 0.002)$ (Ozdemir et al., 2014). Serum ferritin is a hallmark of inadequate iron chelation and vulnerability to develop iron-overloaded complications (Mishra \& Tiwari, 2013). Although, serum ferritin was decreased within three months in the treatment group form $3464.5 \pm 1378.47$ to $3293 \pm 1434.33$. It was not a significant factor for our experiment as its p-value had a higher value of more than 0.005 , as the serum ferritin levels in the treatment group started with higher levels. While in the non-treatment group serum ferritin level was non-significantly increased from $1603.77 \pm 938.91$ to $1886.33 \pm 1352.90$ than non-treatment. Also our study was agreed with previous studies reported that antioxidant and chelation therapy for thalassemia shown efficacy of the decrease in oxidative stress, improvement of antioxidant defense systems, and decrease in iron load (Hamdy et al., 2015). Our results show, positive correlation between TOS and serum ferritin after 3 months with correlation coefficient of 0.382 and with significant p-value of 0.01 . Our results agreed with Naithani et al., 2006, as parameters of oxidative stress were positively correlated with ferritin suggesting the role of excess iron in generating free radicals and the resultant tissue injury. These findings were somewhat compromised by the fact that we used serum ferritin as the sole marker of iron overload, which is an imprecise marker of total body iron burden ( Naithani et al., 2006).

In our study by comparison between 2 groups in serum TOS level before starting treatment, there was no significant difference between them. But by comparison between 2 groups in serum TOS after treatment for 3 months, there was a significant difference as $\mathrm{p}$-value 0.014 . This indicates there was a decrease of oxidants in the treatment group in reverse of the non-treatment group. In our study, there was a 
significant decrease as the p-value of less than 0.001 between TOS before starting treatment and after continuous on treatment for 3 months which indicates the role of NAC in decreasing oxidants. Effect of iron chelator and NAC (as antioxidants) in decrease serum ferritin level which is an indicator of the decrease in the iron load that is responsible for the formation of reactive oxygen species.

In an experimental study, Amer et al., 2008 examined the effects of various oxidants and antioxidants on lipid peroxidation in normal and thalassemia erythrocytes, and they found lipid peroxidation was higher in the thalassemia erythrocytes and these effects were reversed by NAC which coincides with our results and also coincides with Ozdemir et al., $2014[22,18]$

Rodriguez et al., 2018 reported that, increased oxidative stress as well as iron overload in the liver led to use of glutathione and might contribute to decreased levels of glutathione in thalassemia patients. Therefore, NAC use in these patients, by increasing intracellular glutathione, may be expected to have a positive influence on oxidative effects (Rodriguez et al., 2018). In our study, there was a significant increase as p-value 0.002 between TOS before and after 3 months in the nontreatment group which indicates an increase of oxidants could be due to increase in iron load and its effect. Our results are coinciding with the study of Kassab-Chekir et al., 2003 as they reported that there was an increase Oxidant marker which indicates the ongoing oxidative stress in beta-thalassemia, plasma antioxidant defenses were overwhelmed and organs were no longer adequately protected and undergo oxidation (Kassab-Chekir et al., 2003).

Finally, we concluded that in $\beta$-thalassemia major there is an increase in serum oxidant status (TOS) level and we recommend giving NAC as antioxidants to overcome this effect. In this study serum, TOS level decreased in the treatment group besides the improvement of hemoglobin level.

\section{CONCLUSION}

$\mathrm{N}$-acetylcysteine may be effective in reducing serum oxidative stress and increase pre-transfusion $\mathrm{Hb}$ levels in children with $\beta$-thalassemia. 


\section{REFERENCES}

- Cao, A., \& Galanello, R. (2010). Beta thalassemia. Genetics in medicine, 12(2), 61-76 .

- Cappellini, M. D., Cohen, A., Porter, J., Taher, A., \& Viprakasit, V. (Eds.). (2014). Guidelines for the management of transfusion dependent thalassemia (TDT) (pp. 148-9). Nicosia, Cyprus: Thalassaemia International Federation.

- Chan, Y. (2003a). Biostatistics 102: quantitative data-parametric \& nonparametric tests. Blood press, 140(24.08), 79 .

- Chan, Y. (2003b). Biostatistics 103: qualitative data tests of independence. Singapore Med J, 44(10), 498-503 .

- Faintuch, J., Aguilar, P. B., \& Nadalin, W. (1999). Relevance of Nacetylcysteine in clinical practice: fact, myth or consequence?-does added glutamine change the effect? Nutrition, 2(15), 177-179 .

- Hamdy, M. M., Mosallam, D. S., Jamal, A. M., \& Rabie, W. A. (2015). Selenium and Vitamin E as antioxidants in chronic hemolytic anemia: Are they deficient? A case-control study in a group of Egyptian children. Journal of advanced research, 6(6), 1071-1077 .

- Hershko, C. (2010). Pathogenesis and management of iron toxicity in thalassemia. Annals of the New York Academy of Sciences, 1202(1), 1-9 .

- Kassab-Chekir, A., Laradi, S., Ferchichi, S., Khelil, A. H., Feki, M., Amri, F., . . . Miled, A. (2003). Oxidant, antioxidant status and metabolic data in patients with beta thalassemia. Clinica Chimica Acta, 338(1-2), 79-86 .

- Kolnagou, A., Economides, C., Eracleous, E., \& Kontoghiorghes, G. J. (2008). Long term comparative studies in thalassemia patients treated with deferoxamine or a deferoxamine/deferiprone combination. Identification of effective chelation therapy protocols. Hemoglobin, 32(1-2), 41-47 .

- Mishra, A. K., \& Tiwari, A. (2013). Iron overload in beta thalassemia major and intermedia patients. Maedica, 8(4), 328 .

- Muncie Jr, H. L., \& Campbell, J. S. (2009). Alpha and beta thalassemia. American family physician, 80(4), 339-344 .

- Naithani, R., Chandra, J., Bhattacharjee, J., Verma, P., \& Narayan, S. (2006). Peroxidative stress and antioxidant enzymes in children with $\beta$-thalassemia major. Pediatric blood \& cancer, 46(7), 780-785 .

- Origa, R. (2017). $\beta$-thalassemia. Genetics in medicine, 19(6), 609-619 . 
- Ozdemir, Z. C., Koc, A., Aycicek, A., \& Kocyigit, A. (2014). N-acetylcysteine supplementation reduces oxidative stress and DNA damage in children with $\beta$ thalassemia. Hemoglobin, 38(5), 359-364 .

- Patpan, N., Banjerdpongchai, R., Tantiworawit, A., Poofery, J., Komonrit, P., Fanhchaksai, K.,. Norasetthada, L. (2019). The Effect of TransfusionDependent Thalassemia Patient's Serum on Peripheral Blood Mononuclear Cell Viability. Journal of Cell Death, 12, 1179066018823534 .

- Prati, D. (2000). Benefits and complications of regular blood transfusion in patients with beta-thalassemia major. Vox sanguinis, 79(3), 129-137 .

- Rodriguez, B. M., Khouzami, L., Decostre, V., Varnous, S., PekovicVaughan, V., Hutchison, C. J., . . . Muchir, A. (2018). N-acetyl cysteine alleviates oxidative stress and protects mice from dilated cardiomyopathy caused by mutations in nuclear A-type lamins gene. Human molecular genetics, 27(19), 3353-3360 .

- Zastawny, T. H., Altman, S. A., Randers-Eichhorn, L., Madurawe, R., Lumpkin, J. A., Dizdaroglu, M., \& Rao, G. (1995). DNA base modifications and membrane damage in cultured mammalian cells treated with iron ions. Free Radical Biology and Medicine, 18(6), 1013-1022 . 